\title{
pH-Responsive Micellization of Amine-Containing Cationic Diblock Copolymers Prepared by Reversible Addition-Fragmentation Chain Transfer (RAFT) Radical Polymerization
}

\author{
Shin-ichi Yusa,,${ }^{1} \dagger$ Yoshitane KonISHI, ${ }^{1}$ Yoshiro MitsukAmI, ${ }^{2}$ \\ Tohei YAMAMOTO, ${ }^{1}$ and Yotaro MORISHIMA ${ }^{3}$ \\ ${ }^{1}$ Department of Materials Science and Chemistry, Graduate School of Engineering, \\ University of Hyogo, 2167 Shosha, Himeji 671-2201, Japan \\ ${ }^{2}$ Department of Macromolecular Science, Graduate School of Science, Osaka University, \\ 1-1 Machikaneyama-cho, Toyonaka 560-0043, Japan \\ ${ }^{3}$ Faculty of Engineering, Fukui University of Technology, 6-3-1 Gakuen, Fukui 910-8505, Japan
}

(Received December 27, 2004; Accepted March 26, 2005; Published July 15, 2005)

\begin{abstract}
A series of poly([3-(methacryloylamino)propyl]trimethylammonium chloride)-block-poly(2-(diethylamino)ethyl methacrylate) ( MMAPTAC $_{m}-$ DEAEMA $_{n}$ ) of different block lengths were prepared by controlled radical polymerization based on reversible addition-fragmentation chain transfer (RAFT). The diblock copolymers have a narrow distribution in their molecular weights, the ratios of weight- and number-average molecular weights $\left(M_{\mathrm{w}} / M_{\mathrm{n}}\right)$ being in the range of 1.04-1.05. On the basis of ${ }^{1} \mathrm{H}$ NMR, quasielastic light scattering (QELS), static light scattering (SLS), and fluorescence probe data, these diblock copolymers exhibited reversible micelle formation and dissociation in aqueous solutions induced by a $\mathrm{pH}$ change. The hydrodynamic radius $\left(R_{\mathrm{h}}\right)$ and apparent molecular mass for the diblock copolymer increased abruptly near $\mathrm{pH} 7$ with increasing $\mathrm{pH}$, indicative of the micelle formation at basic pHs. Fluorescence spectra for $N$-phenyl-1-naphthylamine (PNA) probes suggested that hydrophobic molecules such as PNA were incorporated into hydrophobic cores in the micelle. PNA probes were released into the bulk water phase when the micelle was dissociated at $\mathrm{pH} \leq 7$. The capture of the fluorescence probe at $\mathrm{pH}>7$ and its release at $\mathrm{pH} \leq 7$ occur reversibly as a result of the formation and dissociation of the micelle upon $\mathrm{pH}$ change.

[DOI 10.1295/polymj.37.480]

KEY WORDS RAFT Radical Polymerization / Block Copolymer / pH-Responsive / Micelle / Living Polymerization / Light Scattering / Fluorescence /
\end{abstract}

Controlled radical polymerization has been shown to be of great use for the synthesis of polymers with well-defined architectures. Several approaches have been reported, which include nitroxide-mediated or alkoxyamine-initiated polymerization (stable free radical polymerization, SFRP technique),${ }^{1-3}$ atom transfer radical polymerization (ATRP), ${ }^{4,5}$ and reversible addition-fragmentation chain transfer (RAFT) radical polymerization. ${ }^{6}$

(Meth)acrylamide-based monomers are important materials for preparation of water-soluble polymers. The controlled radical polymerization of acrylamide derivatives by $\operatorname{SFRP}^{7,8}$ and ATRP $^{9,10}$ is known to be very difficult. On the other hand, RAFT methods seem to be well suited for the polymerization of acrylamide derivatives, e.g., polymerization of $N, N$ dimethylacrylamide (DMA) and $N$-isopropylacrylamide (NIPAM) in organic solvents by the RAFT process have been reported. ${ }^{11,12}$ Recently, McCormick and co-workers reported that various acrylamide derivatives can be polymerized in aqueous media by the RAFT process. ${ }^{13-16}$

Water-soluble stimuli-responsive block copolymers synthesized by controlled radical polymerization have been the subject of increasing interest because they may find practical applications such as nano-size vehicles for drug delivery systems. ${ }^{17-19}$ Several stimuliresponsive block copolymers prepared by controlled radical polymerization have been reported. For example, Laschewky et al. ${ }^{20}$ synthesized water-soluble diblock copolymers of NIPAM and 3-[N-(3-methacrylamidopropyl)- $N, N$-dimethyl]ammoniopropane sulfonate (SPP) by RAFT radical polymerization. These block copolymers exhibit double thermo-responsive behavior in aqueous solutions, i.e., the NIPAM block shows a lower critical solution temperature, whereas the SPP block exhibits an upper critical solution temperature.

In our earlier paper, we reported on a well-defined acrylamide-based diblock copolymer, poly(sodium 2-(acrylamido)-2-methylpropanesulfonate)-block-poly(sodium 6-acrylamidohexanoate) (pNaAMPS-AaH),

To whom correspondence should be addressed (E-mail: yusa@eng.u-hyogo.ac.jp). 
prepared by RAFT radical polymerization. ${ }^{21}$ A similar type block copolymer, diblock copolymer of NaAMPS and 3-acrylamido-3-methylbutanoate (AMBA), has been synthesized by McCormick and co-workers. ${ }^{22}$ These anionic diblock copolymers show $\mathrm{pH}$-responsive properties, i.e., the polymers undergo micellization in acidic solutions, AaH or AMBA forming dehydrated cores, whereas the polymers are molecularly dissolved in basic solutions. Although several examples of anionic block copolymers are known, examples of cationic block copolymers having quaternary ammonium cations are very few. Poly( (ar-vinylbenzyl)trimethylammonium chloride)-block-poly $(N, N$-dimethylvinylbenzylamine) reported by McCormick and co-workers ${ }^{23}$ is one of the example of such cationic block copolymers synthesized by RAFT polymerization. They have reported that poly((ar-vinylbenzyl)trimethylammonium chloride)-block-poly $(N, N$ dimethylvinylbenzylamine) $\left(M_{\mathrm{n}}=51,000 ; M_{\mathrm{w}} / M_{\mathrm{n}}=\right.$ 1.37) forms micelles with $R_{\mathrm{h}}=38.0 \mathrm{~nm}$ at a high $\mathrm{pH}$ whereas it exists as a unimer state with $R_{\mathrm{h}}=8.0$ $\mathrm{nm}$ at a low $\mathrm{pH}$ because the dimethylbenzylamine blocks are also ionized at acidic conditions. Diblock copolymers prepared from two basic monomers of different basicities may be viewed as cationic block copolymers depending on $\mathrm{pH}$. McCormick and coworkers have demonstrated that basic monomers such as $\mathrm{N}$-[3-(dimethylamino)propyl]methacrylamide, ${ }^{24} 2$ vinylpyridine, 4-vinylpyridine, ${ }^{25}$ and $N, N$-dimethylvinylbenzylamine ${ }^{23}$ can be polymerized by RAFT methods. Armes et al. ${ }^{26-29}$ synthesized a series of block copolymers of two basic monomers by group transfer polymerization (GTP) and ATRP, including diblock copolymers of 2-(dimethylamino)ethyl methacrylate (DMAEMA) with 2-(diethylamino)ethyl methacrylate (DEAEMA). DMAEMA homopolymers are soluble in neutral $\mathrm{pH}$ or in acidic solution due to the protonation of the pendent tertiary amine moieties. DEAEMA homopolymer is insoluble in water at neutral pHs, whereas the homopolymer is soluble in acidic aqueous solutions due to the protonation of the pendent tertiary amine moieties. In aqueous solution at $\mathrm{pH} 7-8$, these cationic diblock copolymers form micelles, however at higher $\mathrm{pHs}$, the micelles have a tendency to aggregate and hence precipitate. ${ }^{29}$ At pH 7-8, the DEAEMA block becomes hydrophobic and forms micellar cores, solvated DMAEMA blocks forming micellar coronas. For example, it is reported that a DMAEMA-DEAEMA diblock copolymer $\left(M_{\mathrm{n}}=11,400 ; M_{\mathrm{w}} / M_{\mathrm{n}}=1.10\right)$ forms micelles with ca. $20 \mathrm{~nm}$ in diameter at $\mathrm{pH} 9.5$.

Among cationic monomers, [3-(methacryloylamino)propyl]trimethylammonium chloride (MAPTAC) is a most commonly used commercially available monomer. Polymers of MAPTAC are expected to be of use for various important applications such as tissue engineering materials ${ }^{30}$ and pigment dispersants. ${ }^{31}$ To our knowledge, precisely controlled radical polymerization of MAPTAC is not known to date in the literature. Therefore, our motivation of this work is to examine if MAPTAC can be successfully used for the synthesis of cationic block copolymers by RAFT radical polymerization. Because MAPTAC is a quaternary ammonium moiety substituted on a methacrylamide monomer, an RAFT method, among various controlled radical polymerization methods, may be suitable for synthesis of its polymers in a well-controlled manner.

In this paper, we report on the synthesis of diblock copolymers of MAPTAC and DEAEMA by RAFT polymerization and $\mathrm{pH}$-dependent associative properties of the block copolymers in water. First, we prepared a homopolymer of MAPTAC of well-defined molecular weight by RAFT polymerization and subsequently used the MAPTAC homopolymer obtained as a macro-chain transfer agent (macro-CTA) for block copolymerization with DEAEMA. Above $\mathrm{pH} 7$, DEAEMA blocks are insoluble in water due to deprotonation of tertiary amine moieties ${ }^{26,27,32}$ whereas MAPTAC blocks are soluble in water independent of $\mathrm{pH}$. We investigated $\mathrm{pH}$-responsive micelle formation of poly([3-(methacryloylamino)propyl]trimethylammonium chloride)-block-poly(2-(diethylamino)ethyl methacrylate) $\left(\mathrm{pMAPTAC}_{m}-\mathrm{DEAEMA}_{n}\right) \quad($ Chart 1) in aqueous solution by ${ }^{1} \mathrm{H} \mathrm{NMR}$, quasielastic light scattering (QELS), static light scattering (SLS), and fluorescence probe techniques.

\section{EXPERIMENTAL}

\section{Reagents}

4-Cyanopentanoic acid dithiobenzoate was synthesized according to the method reported by McCormick and co-workers. ${ }^{23}$ [3-(Methacryloylamino)propyl]trimethylammonium chloride (MAPTAC) and 2-(diethylamino)ethyl methacrylate (DEAEMA) were passed through basic alumina columns to remove

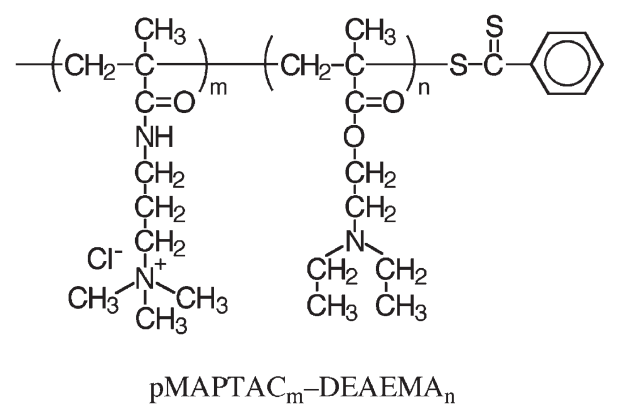

Chart 1. Chemical structure of $\mathrm{pMAPTAC}_{m}-\mathrm{DEAEMA}_{n}$. 
inhibitor. $N$-Phenyl-1-naphthylamine (PNA) was purified by recrystallization from methanol. Water was purified with a Millipore Milli-Q system. Other reagents were used as received.

\section{Preparation of MAPTAC Macro-Chain Transfer Agent (Macro-CTA)}

The preparation of MAPTAC macro-CTA is as follows. MAPTAC $(50.0 \mathrm{~g}, 227 \mathrm{mmol})$ was dissolved in $67.8 \mathrm{~mL}$ of water, and then 4-cyanopentanoic acid dithiobenzoate $\left(421 \mathrm{mg}, 1.51 \mathrm{mmol}\right.$ ) and $4,4^{\prime}$-azobis(4-cyanopentanoic acid) $(84.6 \mathrm{mg}, 0.302 \mathrm{mmol})$ were added to this solution. The mixture was degassed by purging with $\mathrm{Ar}$ gas for $30 \mathrm{~min}$. Polymerization was carried out at $70^{\circ} \mathrm{C}$ for $4 \mathrm{~h}$. The polymerization mixture was poured into a large excess of acetone to precipitate resulting polymer. The polymer was purified by reprecipitating from methanol into a large excess of acetone. The obtained MAPTAC homopolymer could be used as a macro-CTA to prepare block copolymers.

To investigate relationship between polymerization time and conversion, monomer conversion was determined by ${ }^{1} \mathrm{H}$ NMR spectroscopy. Predetermined amounts of MAPTAC, 4-cyanopentanoic acid dithiobenzoate, and initiator were dissolved in $\mathrm{D}_{2} \mathrm{O}$. This solution was transferred to several NMR tubes and deoxygenated by purging with Ar gas for $30 \mathrm{~min}$. After deoxygenation, the cap was sealed and the solutions were heated at $70^{\circ} \mathrm{C}$ in a preheated oil bath for varying lengths of time. The monomer consumption was monitored as a function of polymerization time. Monomer conversion was calculated from a decrease in the area intensity of the vinyl protons at 5.5 and $5.8 \mathrm{ppm}$ in the MAPTAC monomer. Polymerization was terminated by rapid cooling with an ice bath. Gel-permeation chromatography (GPC) for the reaction mixture was measured to estimate the numberaverage molecular weight $\left(M_{\mathrm{n}}\right)$ and molecular weight distribution $\left(M_{\mathrm{w}} / M_{\mathrm{n}}\right)$.

\section{Block Copolymerization}

A typical procedure for block copolymerization is as follows. MAPTAC macro-CTA $(2.93 \mathrm{~g}, 0.21 \mathrm{mmol}$, $\left.M_{\mathrm{n}}=1.40 \times 10^{4} ; M_{\mathrm{w}}=1.44 \times 10^{4} ; M_{\mathrm{w}} / M_{\mathrm{n}}=1.03\right)$, DEAEMA $(2.41 \mathrm{~g}, 13.0 \mathrm{mmol})$, and $4,4^{\prime}$-azobis(4cyanopentanoic acid) $(11.8 \mathrm{mg}, 0.042 \mathrm{mmol})$ were dissolved in $20.5 \mathrm{~mL}$ of water. The solution $\mathrm{pH}$ was adjusted to 6.5 to add $\mathrm{HCl}(6.0 \mathrm{M})$ to the solution. The solution was deoxygenated by purging with $\mathrm{Ar}$ gas for $30 \mathrm{~min}$. Block copolymerization was carried out at $70^{\circ} \mathrm{C}$ for $4 \mathrm{~h}$. The diblock copolymer was purified by dialysis against pure water for a week, changing the pure water twice a day. The diblock copolymer was recovered by a freeze-drying technique.
To investigate relationship between polymerization time and conversion, predetermined amounts of DEAEMA, MAPTAC macro-CTA, and initiator were dissolved in $\mathrm{D}_{2} \mathrm{O}$ and $\mathrm{pH}$ was adjusted to 6.5 to add DCl. This stock solution was transferred to several NMR tubes and deoxygenated by purging with $\mathrm{Ar}$ gas for $30 \mathrm{~min}$. After deoxygenation, the cap was sealed and the solutions were heated at $70^{\circ} \mathrm{C}$ in an oil bath for varying lengths of time. Conversion and $M_{\mathrm{n}}$ were characterized by ${ }^{1} \mathrm{H}$ NMR and GPC, respectively.

\section{Measurements}

Gel-permeation chromatography (GPC) analysis was performed using a refractive index (RI) detector equipped with a Shodex Ohpak SB-804 HQ column working at $40^{\circ} \mathrm{C}$ under a flow rate of $1 \mathrm{~mL} / \mathrm{min}$. A $0.3-\mathrm{M} \mathrm{Na}_{2} \mathrm{SO}_{4}$ aqueous solution containing a $0.5-\mathrm{M}$ acetic acid was used as eluent. Molecular weights of the sample polymers were calibrated with standard poly(2-vyniypyridine) samples of 6 different molecular weights ranging from $5.70 \times 10^{3}$ to $3.16 \times 10^{5}$.

${ }^{1} \mathrm{H}$ NMR spectra were obtained with a Bruker DRX-500 spectrometer operating at $500 \mathrm{MHz}$. Chemical shifts were determined by using 3-(trimethylsilyl)propionic-2,2,3,3- $d_{4}$ acid as an internal reference. The sample solutions of the diblock copolymers at $C_{\mathrm{p}}=10 \mathrm{~g} / \mathrm{L}$ for ${ }^{1} \mathrm{H}$ NMR measurements were prepared in $\mathrm{D}_{2} \mathrm{O}$ containing $0.1 \mathrm{M} \mathrm{NaCl}$, and $\mathrm{pD}$ was adjusted with a $\mathrm{D}_{2} \mathrm{O}$ solution of $\mathrm{NaOD}$ or $\mathrm{DCl}$. The final $\mathrm{pD}$ value was determined from the relation $\mathrm{pD}=\mathrm{pH}+0.4 .^{33}$

Quasielastic light scattering (QELS) data were obtained at $25^{\circ} \mathrm{C}$ with an Otsuka Electronics Photal DLS-7000DL light scattering spectrometer equipped with an ALV-5000E multi- $\tau$ digital time correlator. $\mathrm{An} \mathrm{Ar}^{+}$laser $(30.0 \mathrm{~mW}$ at $488 \mathrm{~nm}$ ) was used as a light source. Sample solutions for QELS measurements were filtered with a $0.2 \mu \mathrm{m}$ pore size membrane filter. To obtain the relaxation time distribution, $\tau A(\tau)$, the inverse Laplace transform (ILT) analysis was performed using the algorithm REPES. ${ }^{34,35}$

$$
g^{(1)}(t)=\int \tau A(\tau) \exp (-t / \tau) \mathrm{d} \ln \tau
$$

Here, $\tau$ is the relaxation time and $g^{(1)}(t)$ is the normalized autocorrelation function. The relaxation time distributions are given as a $\tau A(\tau)$ versus $\log \tau$ profile with an equal area. The average translational diffusion coefficient $(D)$ is calculated from $D=\Gamma / q^{2}$, where $\Gamma$ is the inverse of $\tau$, i.e., the average relaxation rate, and $q=(4 \pi n / \lambda) \sin (\theta / 2)$ with $n$ being the refractive index of solvent, $\lambda$ being the wavelength $(=488 \mathrm{~nm})$, and $\theta$ being the scattering angle. The intensity-average hydrodynamic radius $\left(R_{\mathrm{h}}\right)$ is calculated using the Einstein-Stokes relation $R_{\mathrm{h}}=k_{\mathrm{B}} T / 6 \pi \eta D$, where $k_{\mathrm{B}}$ 
is Boltzmann's constant, $T$ is the absolute temperature, and $\eta$ is the solvent viscosity.

Static light scattering (SLS) measurements were performed at $25^{\circ} \mathrm{C}$ with an Otsuka Electronics Photal DLS-7000DL light scattering spectrometer equipped with an $\mathrm{He}-\mathrm{Ne}$ laser $(10 \mathrm{~mW}$ at $632.8 \mathrm{~nm})$. Sample solutions for SLS measurements were filtered with a $0.2 \mu \mathrm{m}$ pore size membrane filter. The weight-average molecular weight $\left(M_{\mathrm{w}}\right)$ and the second virial coefficient $\left(A_{2}\right)$ values were estimated from the relation ${ }^{36}$

$$
\frac{K C_{\mathrm{p}}}{R_{\theta}}=\frac{1}{M_{\mathrm{w}}}\left(1+\frac{1}{3}\left\langle R_{\mathrm{g}}^{2}\right\rangle q^{2}\right)+2 A_{2} C_{\mathrm{p}}
$$

where $C_{\mathrm{p}}$ is the polymer concentration, $R_{\theta}$ is the Rayleigh ratio, $R_{\mathrm{g}}$ is the $z$-average radius of gyration, and $K=4 \pi^{2} n^{2}\left(\mathrm{~d} n / \mathrm{d} C_{\mathrm{p}}\right) 2 / N_{\mathrm{A}} \lambda^{4}$ with $\mathrm{d} n / \mathrm{d} C_{\mathrm{p}}$ being the refractive index increment against $C_{\mathrm{p}}$ and $N_{\mathrm{A}}$ being Avogadro's number. By measuring $R_{\theta}$ for a set of $C_{\mathrm{p}}$ and $\theta$, values of $M_{\mathrm{w}}$ and $A_{2}$ were estimated from Zimm plots. Toluene was used for the calibration of the instrument. Values of $\mathrm{d} n / \mathrm{d} C_{\mathrm{p}}$ were determined with an Otsuka Electronics Photal DRM-1020 differential refractometer.

Fluorescence spectra were recorded on a Hitachi F-2500 fluorescence spectrophotometer. A $0.1-\mathrm{M}$ $\mathrm{NaCl}$ aqueous stock solution of PNA $\left(1.0 \times 10^{-6} \mathrm{M}\right)$ was prepared. For PNA fluorescence measurements, the sample solutions were excited at $340 \mathrm{~nm}$, and excitation and emission slit widths were maintained at 20 and $5.0 \mathrm{~nm}$, respectively.

\section{RESULTS AND DISCUSSION}

\section{Synthesis of MAPTAC Macro-CTA}

In Figure 1a, a time-conversion plot is depicted along with the pseudo first-order kinetic plot for the RAFT polymerization of MAPTAC in $\mathrm{D}_{2} \mathrm{O}$ under an $\mathrm{Ar}$ atmosphere. The linear increase of the first-order plot indicates that the reaction is first-order and the polymer radical concentration remains essentially constant during the polymerization. A GPC elution curve measured for each plot in Figure 1a is shown in Figure 1b. The molecular mass increases progressively with polymerization time, and the GPC elution bands are unimodal with no sign of contamination from uncontrolled polymerization. The GPC results shown in Figure 1c indicate that $M_{\mathrm{n}}$ of MAPTAC homopolymer increases with conversion while $M_{\mathrm{w}} /$ $M_{\mathrm{n}}$ remains nearly constant at 1.04-1.05. Apparent deviation of the conversion- $M_{\mathrm{n}}$ plot from a strait line passing through the origin may be due to an unexpected interaction of the GPC column and the polymer and a difference between the obtained polymer and standard poly(2-vyniypyridine) samples. In a control experiment, MAPTAC homopolymer was prepared
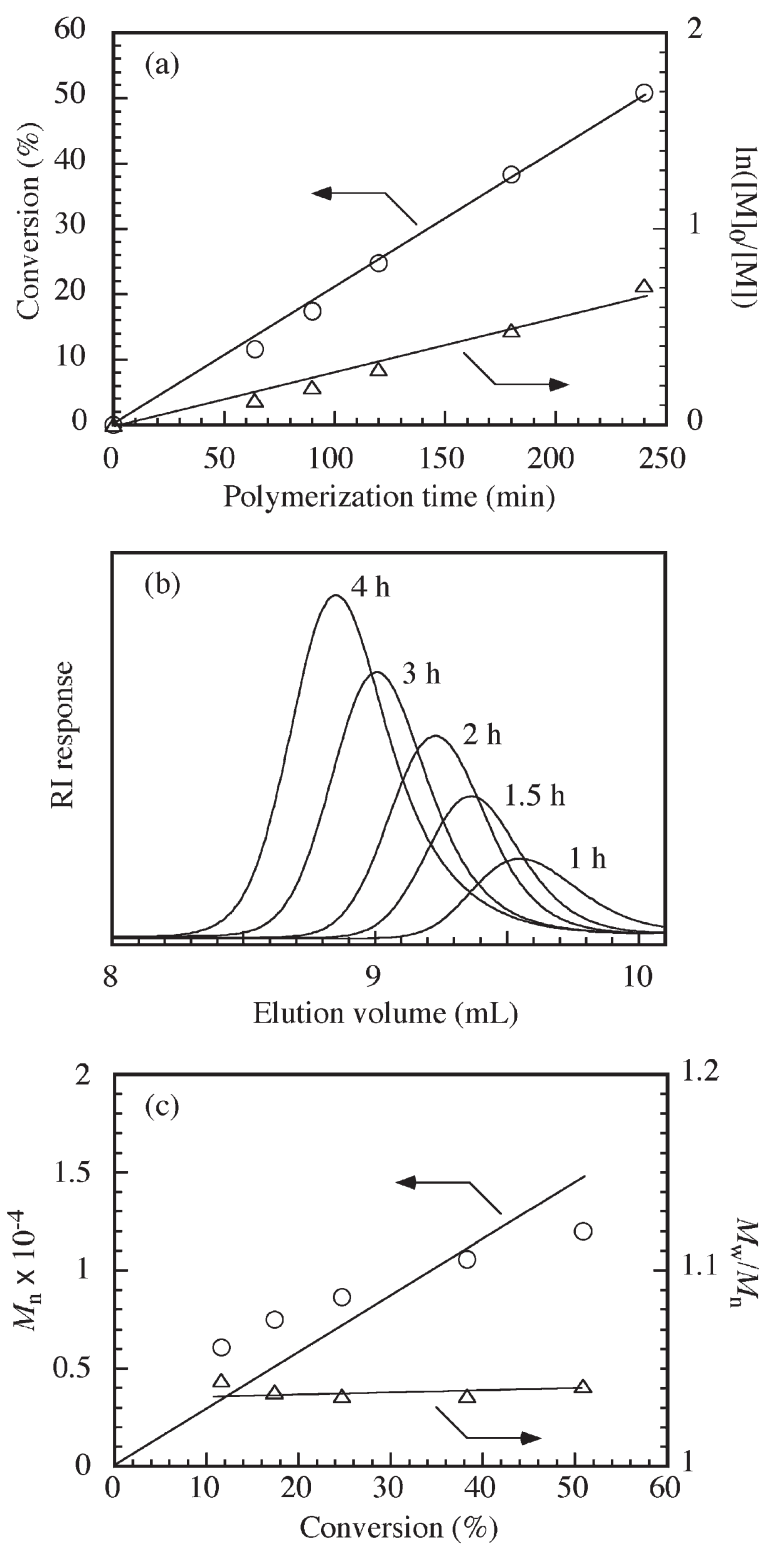

Figure 1. (a) Time-conversion $(\bigcirc)$ and the pseudo first-order kinetic plots $(\triangle)$ for the polymerization of MAPTAC in the presence of 4-cyanopentanoic acid dithiobenzoate in $\mathrm{D}_{2} \mathrm{O}$ at $70^{\circ} \mathrm{C}$. (b) Evolution of GPC elusion curves during the synthesis of MAPTAC homopolymer with polymerization time. (c) Dependence of $M_{\mathrm{n}}(\bigcirc)$ and $M_{\mathrm{w}} / M_{\mathrm{n}}(\triangle)$ on the monomer conversion in the polymerization of MAPTAC.

by conventional free radical polymerization under the same conditions as those for the RAFT polymerization except 4-cyanopentanoic acid dithiobenzoate (CTA) was not added. The reaction mixture became highly viscous within $30 \mathrm{~min}$. The values of $M_{\mathrm{n}}$ and $M_{\mathrm{w}} / M_{\mathrm{n}}$ for the MAPTAC homopolymer obtained in the controlled experiment were found to be $2.11 \times$ $10^{5}$ and 12.0 , respectively. On the other hand, the values of $M_{\mathrm{n}}$ and $M_{\mathrm{w}} / M_{\mathrm{n}}$ for the purified homopolymer prepared by the RAFT process at a conversion of $41.2 \%$ are $1.40 \times 10^{4}$ and 1.03 , respectively. These results indicate that the polymerization of MAPTAC 
Table I. Molecular weights and compositions of the block copolymers

\begin{tabular}{ccccc}
\hline Sample code & $\begin{array}{c}\text { DP of MAPTAC } \\
\text { block }^{\mathrm{a}}\end{array}$ & $\begin{array}{c}\text { DP of DEAEMA } \\
\text { block }^{\mathrm{b}}\end{array}$ & $M_{\mathrm{n}, \mathrm{GPC}} \times 10^{-4}$ & $M_{\mathrm{w}} / M_{\mathrm{n}}$ \\
\hline pMAPTAC $_{62}-$ DEAEMA $_{11}$ & 62 & 11 & 1.48 & 1.04 \\
pMAPTAC $_{62}-$ DEAEMA $_{28}$ & 62 & 28 & 1.61 & 1.04 \\
pMAPTAC $_{62}-$ DEAEMA $_{53}$ & 62 & 53 & 1.81 & 1.05 \\
\hline
\end{tabular}

${ }^{\text {a }}$ Degree of polymerization (DP) determined by GPC. ${ }^{b} \mathrm{DP}$ estimated by ${ }^{1} \mathrm{H}$ NMR.

by the RAFT process follows a virtually "living" polymerization mechanism.

\section{Synthesis of Diblock Copolymers of MAPTAC and DEAEMA}

We performed polymerization of DEAEMA in the presence of MAPTAC macro-CTA $\left(M_{\mathrm{n}}=1.40 \times\right.$ $\left.10^{4} ; M_{\mathrm{w}}=1.44 \times 10^{4} ; M_{\mathrm{w}} / M_{\mathrm{n}}=1.03\right)$ in $\mathrm{D}_{2} \mathrm{O}$ at $\mathrm{pH}$ 6.5. Because the DEAEMA monomer is basic, $\mathrm{pH}$ of its aqueous solution was adjusted to 6.5 to avoid undesirable side reactions including hydrolysis of macro-CTA. ${ }^{37}$ Thiocarbonyl compounds, such as CTA used in the present work, are known to be unstable toward hydrolysis. ${ }^{38}$ Figure 2 a shows a timeconversion and the pseudo first-order kinetic plots for the polymerization of DEAEMA in the presence of MAPTAC macro-CTA. A high conversion of DEAEMA ( $>97 \%$ ) was reached at a polymerization time of $240 \mathrm{~min}$. It should be noted here that a conversion of $\sim 51 \%$ is obtained for the homopolymerization of MAPTAC at the same polymerization time (Figure 1a). It is known that the polymerization rate and conversion of RAFT radical polymerization are sensitive to a combination of CTA and monomer. The first-order plots for the copolymerization of DEAEMA are approximately fitted to a straight line.

In Figure 2b, a GPC elusion curve for the MAPTAC-DEAEMA diblock copolymer is compared with that for the MAPTAC macro-CTA before copolymerization. A clear increase in the molecular weight occurs upon polymerization of DEAEMA in the presence of MAPTAC macro-CTA. Neither a new peak nor a shoulder due to DEAEMA homopolymer formed by homopolymerization was recognized. The GPC results shown in Figure $2 \mathrm{c}$ indicate that $M_{\mathrm{n}}$ increases linearly with conversion. Taken together with a narrow $M_{\mathrm{w}} / M_{\mathrm{n}}$, this result indicates that the polymerization is well controlled.

We prepared a series of diblock copolymers of different DEAEMA bock lengths using the same MAPTAC macro-CTA $\left(M_{\mathrm{n}}=1.40 \times 10^{4} ; \quad M_{\mathrm{w}}=\right.$ $\left.1.44 \times 10^{4} ; M_{\mathrm{w}} / M_{\mathrm{n}}=1.03\right)$. The degrees of polymerization (DP) of MAPTAC and DEAEMA blocks were calculated from GPC and ${ }^{1} \mathrm{H}$ NMR, respectively. Table I lists some molecular characteristics of these diblock copolymers.
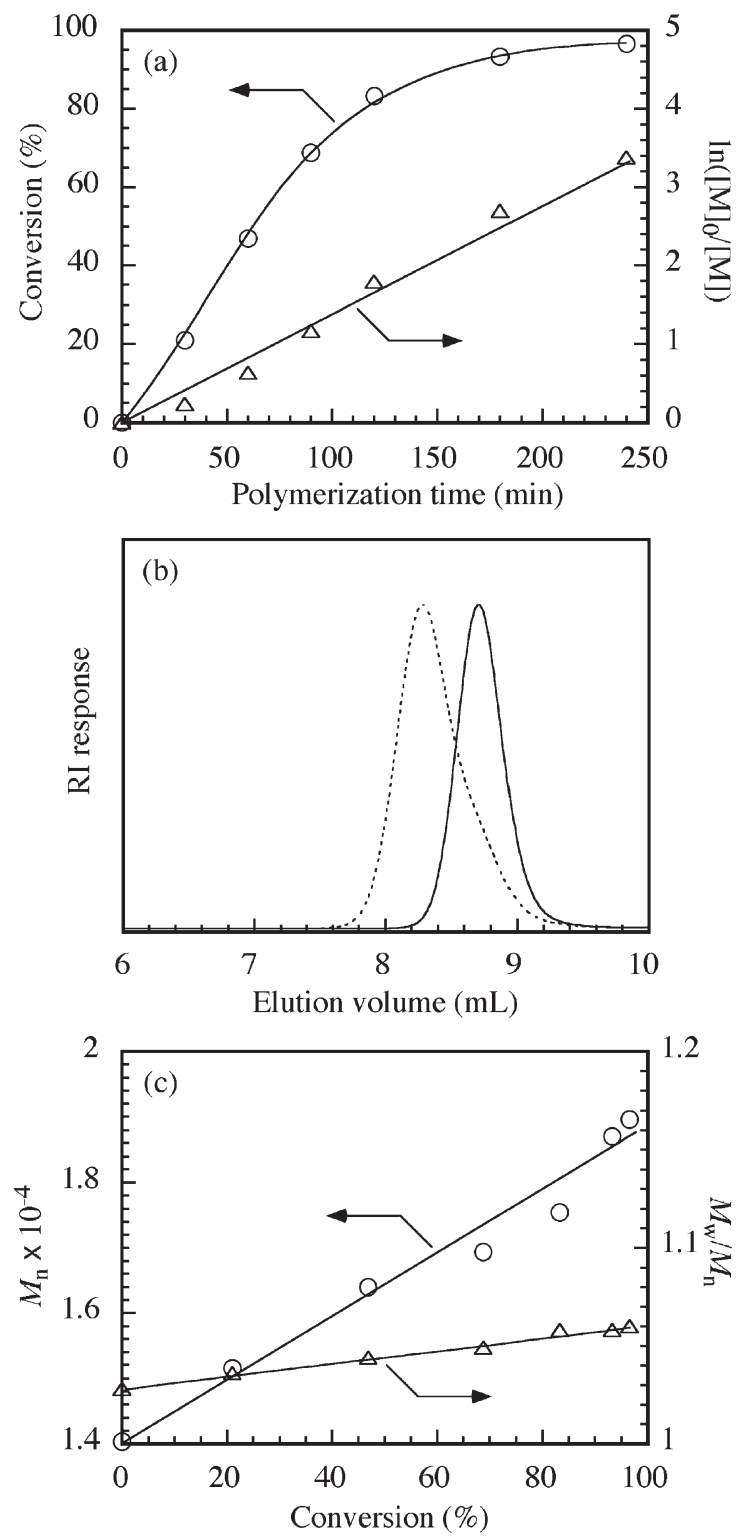

Figure 2. (a) Time-conversion $(\bigcirc)$ and the pseudo first-order kinetic plots $(\triangle)$ for the polymerization of DEAEMA in the presence of MAPTAC macro-CTA in $\mathrm{D}_{2} \mathrm{O}$ at $70^{\circ} \mathrm{C}$. (b) GPC elution curves for a sample of MAPTAC macro-CTA $\left(M_{\mathrm{n}}=1.40 \times 10^{4}\right.$; $\left.M_{\mathrm{w}} / M_{\mathrm{n}}=1.03\right)(-)$ and the corresponding diblock copolymer of MAPTAC and DEAEMA $\left(M_{\mathrm{n}}=1.90 \times 10^{4} ; M_{\mathrm{w}} / M_{\mathrm{n}}=1.06\right)$ (-- -). (c) Dependence of $M_{\mathrm{n}}(\bigcirc)$ and $M_{\mathrm{w}} / M_{\mathrm{n}}(\triangle)$ on the monomer conversion in the polymerization of DEAEMA in the presence of MAPTAC macro-CTA. 


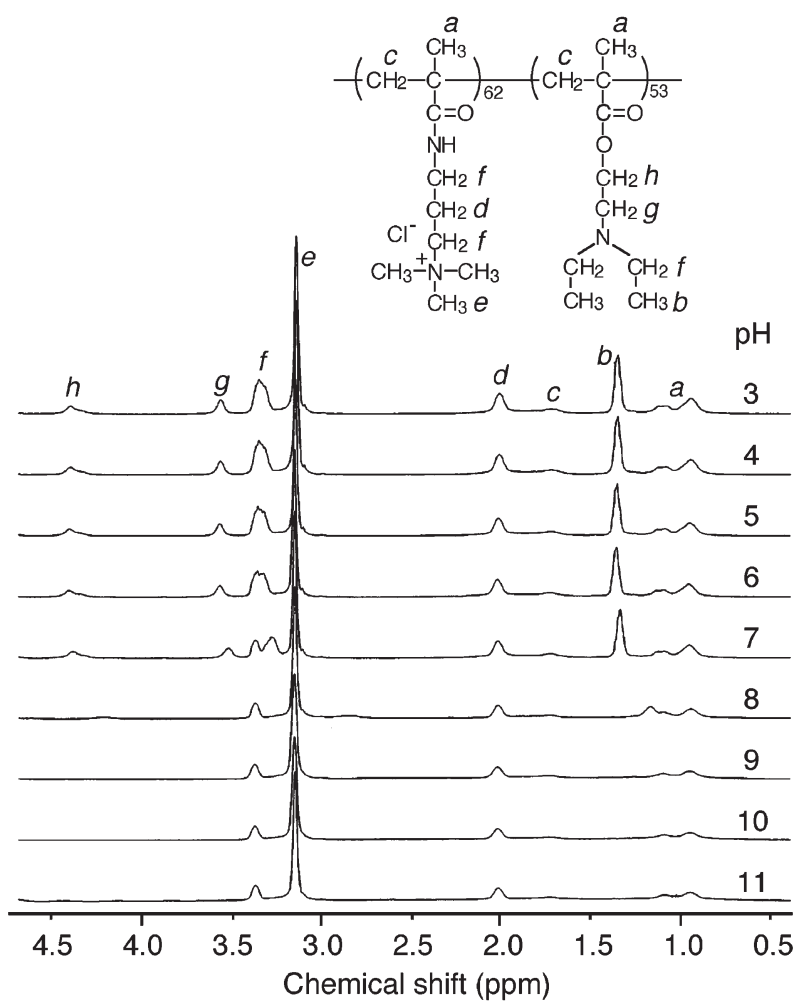

Figure 3. ${ }^{1} \mathrm{H}$ NMR spectra for pMAPTAC $_{62}-$ DEAEMA $_{53}$ at $C_{\mathrm{p}}=10 \mathrm{~g} / \mathrm{L}$ in $\mathrm{D}_{2} \mathrm{O}$ containing $0.1 \mathrm{M} \mathrm{NaCl}$ at varying $\mathrm{pHs}$.

\section{${ }^{1} H N M R$}

Figure 3 shows the ${ }^{1} \mathrm{H}$ NMR spectra for pMAPTAC $_{62}-$ DEAEMA $_{53}$ (Chart 1), where the subscript represents the degree of polymerization of each block, measured at $C_{\mathrm{p}}=10 \mathrm{~g} / \mathrm{L}$ in $0.1 \mathrm{M} \mathrm{NaCl}$ containing $\mathrm{D}_{2} \mathrm{O}$ at different $\mathrm{pH}$. At $\mathrm{pH} 3$, the diblock copolymer chains are fully protonated and hence solvated, and all the signals expected for each block were observed. Under these conditions, the diblock copolymer is molecularly dissolved in water, i.e., a "unimer" state. The resonance bands in the $0.8-1.1 \mathrm{ppm}$ region and at $1.8 \mathrm{ppm}$ are attributed to the $\alpha$-methyl protons and the main chain methylene protons, respectively. The composition of the diblock copolymer was determined from the intensity ratio of the resonance bands due to the methyl protons in the side chain of the DEAEMA block at $1.3 \mathrm{ppm}$ and the trimethyl amino protons of the MAPTAC block at $3.2 \mathrm{ppm}$ in $\mathrm{D}_{2} \mathrm{O}$ at $\mathrm{pH}$ 3. Progressive deprotonation of DEAEMA units leads to a small upfield shift in the signals due to the pendent diethylaminoethyl groups at $\mathrm{pH} \leq 7 .^{29}$ The DEAEMA homopolymer is weakly basic with a $\mathrm{p} K_{\mathrm{a}}$ of $c a .7 .3{ }^{39}$ Above $\mathrm{pH} 8$, the resonance peaks corresponding to the DEAEMA block disappear completely. The disappearance of the resonance signals assigned to the DEAEMA block implies poor solvation and reduced mobility of the DEAEMA block. Considering the chemical structure of the block copolymer, one can expect that the polymer is likely to form a core-corona type polymer micelle with deprotonated DEAEMA blocks forming a core and permanently charged MAPTAC blocks forming a corona. When the solution $\mathrm{pH}$ was increased from 3 to 11 and subsequently decreased back from 11 to 3 , the original NMR spectrum was observed. Therefore, this $\mathrm{pH}$-induced association seems to be reversible. Similar observations were reported by Armes et al. ${ }^{32}$ for the poly(ethylene oxide) (PEO)-DEAEMA diblock copolymer micelle that includes fully dehydrated DEAEMA cores at $\mathrm{pH} 9$.

\section{Light Scattering}

The $\mathrm{pH}$-induced micellization of the diblock copolymer in aqueous solution was confirmed by QELS measurements. The intensity-average hydrodynamic radius $\left(R_{\mathrm{h}}\right)$ for the diblock copolymer was measured in $0.1 \mathrm{M} \mathrm{NaCl}$ aqueous solutions. The plots of the inverse of average relaxation time $(\Gamma)$ and the square of magnitude of the scattering vector $\left(q^{2}\right)$ for the diblock copolymers at $\mathrm{pH} 4$ and 10 are linear relationship passing through the origin was obtained (data not shown). Thus, the measured relaxation time was predominantly attributed to a diffusive mode. QELS measurements were performed at a fixed scattering angle of $90^{\circ}$.

Figure 4 compares relaxation time distributions for pMAPTAC $_{62}-$ DEAEMA $_{53}$ at $\mathrm{pH} 4$ and 10 in $0.1 \mathrm{M}$ $\mathrm{NaCl}$ aqueous solutions. The distributions are unimodal at $\mathrm{pH} 4$ and 10 with different relaxation times. The fast relaxation mode at $\mathrm{pH} 4$ is attributed to a unimer with $R_{\mathrm{h}}=4.9 \mathrm{~nm}$ whereas the slow relaxation time at $\mathrm{pH} 10$ to the polymer aggregates with $R_{\mathrm{h}}=13 \mathrm{~nm}$. This observation suggests the formation of micelles with a DEAEMA core and MAPTAC corona.

Figure 5a compares variation of scattering intensities for the aqueous solutions of the diblock copolymers of different lengths of the DEAEMA block plotted as a function of $\mathrm{pH}$. The scattering intensities

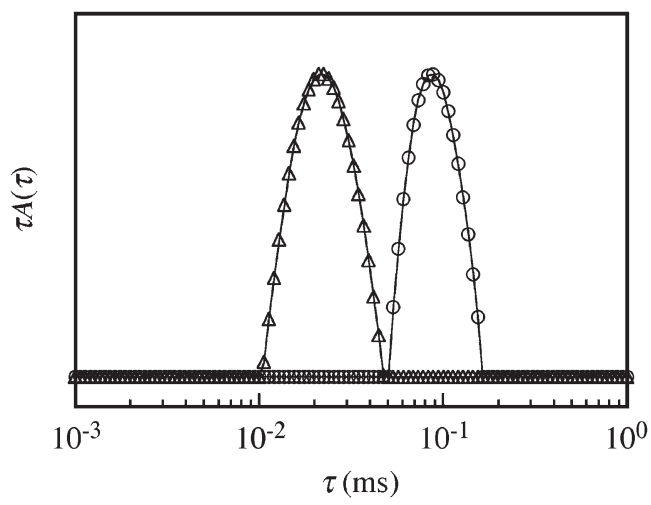

Figure 4. Typical examples of QELS relaxation time distributions for pMAPTAC $_{62}-$ DEAEMA $_{53}$ at $C_{\mathrm{p}}=10 \mathrm{~g} / \mathrm{L}$ in $0.1 \mathrm{M}$ $\mathrm{NaCl}$ aqueous solutions at $\mathrm{pH} 4(\triangle)$ and $10(\bigcirc)$. 

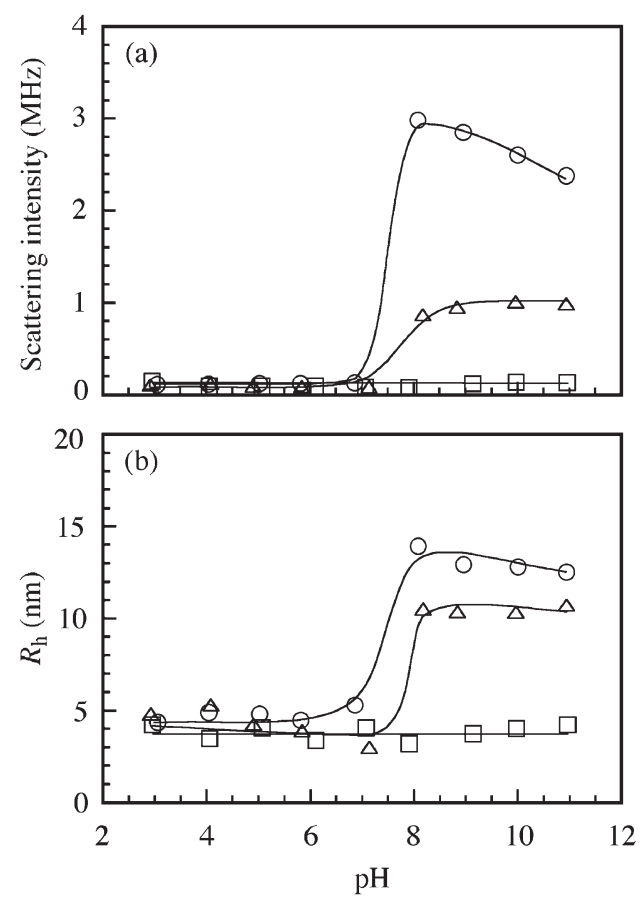

Figure 5. (a) Scattering intensity and (b) intensity-average hydrodynamic radius $\left(R_{\mathrm{h}}\right)$ at $C_{\mathrm{p}}=10 \mathrm{~g} / \mathrm{L}$ for pMAPTAC $_{62}-$ DEAEMA $_{53}(\bigcirc), \quad$ pMAPTAC $_{62}-$ DEAEMA $_{28} \quad(\triangle), \quad$ and pMAPTAC $_{62}-$ DEAEMA $_{11}(\square)$ as a function of $\mathrm{pH}$ in $0.1 \mathrm{M} \mathrm{NaCl}$ aqueous solutions at $25^{\circ} \mathrm{C}$.

for pMAPTAC $_{62}-$ DEAEMA $_{53}$ and pMAPTAC $_{62}-$ DEAEMA $_{28}$ increase drastically as the solution $\mathrm{pH}$ is increased from 7 to 8 . This is a manifestation that polymer micelles are formed at $\mathrm{pH}>7$. On the other hand, the scattering intensity for pMAPTAC $_{62}-$ DEAEMA $_{11}$ remains essentially unchanged upon a $\mathrm{pH}$ change from $\mathrm{pH} 3$ to 11 , suggesting that pMAPTAC $_{62}-$ DEAEMA $_{11}$ does not form polymer micelle presumably because the hydrophobicity of the shorter deprotonated DEAEMA blocks is not sufficient enough to form a micelle core.

Figure $5 \mathrm{~b}$ shows $R_{\mathrm{h}}$ for the diblock copolymers plotted against $\mathrm{pH}$. Below $\mathrm{pH} 7$, the $R_{\mathrm{h}}$ values for the diblock copolymers are on the order of 3-5 nm, suggesting that all the polymers exist in a unimer state. Upon increase in $\mathrm{pH}$, the $R_{\mathrm{h}}$ values for pMAPTAC $_{62}-$ DEAEMA $_{53}$ and pMAPTAC $_{62}-$ DEAEMA $_{28}$ commence to increase at $\mathrm{pH}>7$. This indicates the formation of polymer micelles under basic conditions. The $R_{\mathrm{h}}$ values for $\mathrm{pMAPTAC}_{62}$-DEAEMA $_{53}$ micelles are slightly larger than those for the pMAPTAC ${ }_{62}-$ DEAEMA $_{28}$ micelles. In the case of pMAPTAC ${ }_{62}-$ DEAEMA $_{11}$, the $R_{\mathrm{h}}$ values are constant around $4 \mathrm{~nm}$ in the whole range of $\mathrm{pH}$ examined, which indicates that there is no interpolymer association. The $\mathrm{pH}$ dependence of $R_{\mathrm{h}}$ is consistent with that of the scattering intensity. The $R_{\mathrm{h}}$ values for the diblock copolymers at $\mathrm{pH} 4$ and 10 are summarized in Table II. When $\mathrm{pH}$
Table II. Light scattering data for the block copolymers

\begin{tabular}{ccccc}
\hline Sample code & $\mathrm{pH}$ & $\begin{array}{c}R_{\mathrm{h}} \\
(\mathrm{nm})\end{array}$ & $M_{\mathrm{w}, \mathrm{SLS}} \times 10^{-4}$ & $\begin{array}{c}A_{2} \times 10^{3} \\
(\mathrm{~mol} \mathrm{~mL} \mathrm{~g})\end{array}$ \\
\hline pMAPTAC $_{62}-$ DEAEMA $_{11}$ & 4 & 3.48 & 0.83 & 5.88 \\
& 10 & 4.02 & 1.17 & 1.82 \\
pMAPTAC $_{62}-$ DEAEMA $_{28}$ & 4 & 5.28 & 1.14 & 5.68 \\
& 10 & 10.3 & 11.7 & 0.37 \\
pMAPTAC $_{62}-$ DEAEMA $_{53}$ & 4 & 4.88 & 1.09 & 4.39 \\
& 10 & 12.8 & 30.1 & 0.09 \\
\hline
\end{tabular}

Table III. Characteristics of pMAPTAC-DEAEMA block copolymer micelles

\begin{tabular}{cccc}
\hline Sample code & $\begin{array}{c}R_{\mathrm{c}} \\
(\mathrm{nm})\end{array}$ & $\begin{array}{c}L \\
(\mathrm{~nm})\end{array}$ & $N_{\mathrm{agg}}$ \\
\hline pMAPTAC $_{62}-$ DEAEMA $_{28}$ & 2.39 & 7.91 & 10.3 \\
pMAPTAC $_{62}-$ DEAEMA $_{53}$ & 3.80 & 9.00 & 27.6 \\
\hline
\end{tabular}

was increased from 3 to 11 and subsequently decreased back to 3, completely reversible changes in the $R_{\mathrm{h}}$ values without hysteresis were observed.

The $R_{\mathrm{h}}$ value is the sum of the radius of the core $\left(R_{\mathrm{c}}\right)$ and the corona thickness $(L)$. The $R_{\mathrm{c}}$ can be calculated from the eq $3^{40,41}$

$R_{\mathrm{c}}=\left[3 M_{\mathrm{w}, \text { mic }} w_{\text {DEAEMA }} /\left(4 \pi N_{\mathrm{A}} \rho_{\text {DEAEMA }} \Phi_{\text {DEAEMA }}\right)\right]^{1 / 3}$

where $M_{\mathrm{w}, \text { mic }}$ is the weight-average molecular weight of micelle, $w_{\text {DEAEMA }}$ is the weight fraction of DEAEMA in the diblock copolymer, $N_{\mathrm{A}}$ is Avogadro's number, $\rho_{\text {DEAEMA }}$ is the density of DEAEMA, and $\Phi_{\text {DEAEMA }}$ is the volume fraction of DEAEMA in the core of micelle. Using the values of $\rho_{\text {DEAEMA }}=0.92 \mathrm{~g} / \mathrm{mL}$ which is bulk density of DEAEMA monomer and $\Phi_{\text {DEAEMA }}=1, R_{\mathrm{c}}$ can be determined. The $L$ value can also be calculated from the difference of $R_{\mathrm{h}}$ and $R_{\mathrm{c}}$. These $R_{\mathrm{c}}$ and $L$ values for pMAPTAC $_{62}-$ DEAEMA $_{53}$ and pMAPTAC $_{62}-$ DEAEMA $_{28}$ micelles are summarized in Table III.

Typical examples of Zimm plots for pMAPTAC ${ }_{62}-$ DEAEMA $_{53}$ obtained by SLS measurements in $0.1 \mathrm{M}$ $\mathrm{NaCl}$ aqueous solutions at $\mathrm{pH} 4$ and 10 are shown in Figure 6. The apparent values of $M_{\mathrm{w}}$ for the unimer and micelle were estimated by extrapolation of $C_{\mathrm{p}}$ and $\theta$ to zero, and $A_{2}$ was estimated from the concentration dependence in the Zimm plots. Unfortunately, $R_{\mathrm{g}}$ values for the unimer and micelle are too small to be determined by Zimm plots, i.e., $R_{\mathrm{g}}<10 \mathrm{~nm}$. The $M_{\mathrm{w}}$ and $A_{2}$ values determined from the Zimm plots for the diblock copolymers at $\mathrm{pH} 4$ and 10 are presented in Table II. The $M_{\mathrm{w}}$ value for pMAPTAC $62-$ DEAEMA $_{11}$ at $\mathrm{pH} 10$ is near the value estimated at $\mathrm{pH} 4$, which indicates that pMAPTAC $_{62}-$ DEAEMA $_{11}$ does not form micelles at $\mathrm{pH} 10$. The numbers of polymer chains consisting of one micelle (i.e., aggre- 


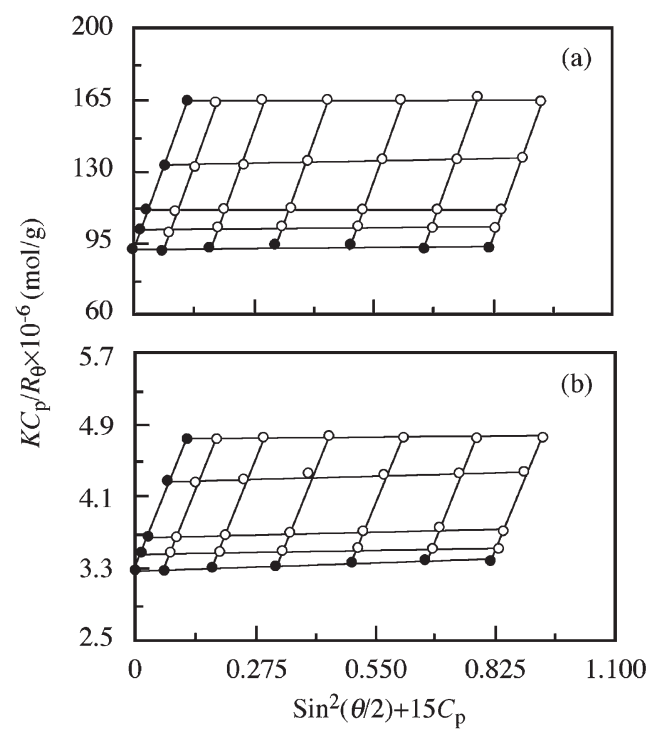

Figure 6. Zimm plots analysis for pMAPTAC ${ }_{62}-$ DEAEMA $_{53}$ in $0.1 \mathrm{M} \mathrm{NaCl}$ aqueous solutions at $\mathrm{pH} 4$ (a) and 10 (b). The polymer concentration was varied from 1.0 to $8.0 \mathrm{~g} / \mathrm{L}$ at angles from $30^{\circ}$ to $130^{\circ}$ with a $20^{\circ}$ increment.

gation numbers) $\left(N_{\mathrm{agg}}\right)$ for pMAPTAC $62-$ DEAEMA $_{53}$ and pMAPTAC $_{62}-$ DEAEMA $_{28}$ micelles may be calculated from the ratio of the $M_{\mathrm{W}}$ values for the micelle at $\mathrm{pH} 10$ and unimer at $\mathrm{pH} 4$ estimated from SLS data (Table III). The values of $M_{\mathrm{w}}$ and $N_{\text {agg }}$ for the block copolymer micelles increase with increasing DEAEMA block length.

\section{Fluorescence}

It is known that steady-state fluorescence spectra of pyrene provides information about microenvironmental polarity. ${ }^{42} \mathrm{We}$ attempted to measure pyrene fluorescence in the presence of our block copolymers, however, pyrene fluorescence was found to be quenched significantly with increasing $C_{\mathrm{p}}$. It was confirmed, in a separate experiment, that pyrene fluorescence was not quenched by the homopolymer of MAPTAC or DEAEMA prepared by ordinary radical polymerization. Therefore, it can be concluded that the dithiobenzoate group in the polymer chain end of the diblock copolymer prepared by the RAFT process is responsible for the fluorescence quenching. Studies of the pyrene fluorescence quenching by polymers synthesized by RAFT are currently under way in our laboratory, and some results of these studies will be reported elsewhere in due time.

It is known that the fluorescence of $\mathrm{N}$-phenyl-1naphthylamine (PNA) is sensitive to its surrounding polarity. ${ }^{43-45}$ As the emission maximum shifts toward shorter wavelengths according to a decrease in the polarity, PNA can be used as a probe for micro polarity. We confirmed that PNA fluorescence was not quenched by 4-cyanopentanoic acid dithiobenzoate

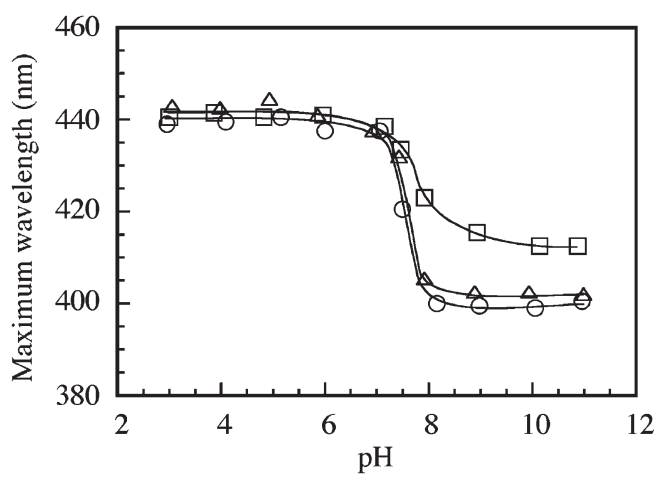

Figure 7. Emission maxima in PNA fluorescence spectra plotted as a function of $\mathrm{pH}$ in the presence of pMAPTAC $_{62}-$ DEAEMA $_{53}(\bigcirc), \quad$ pMAPTAC $_{62}-$ DEAEMA $_{28}(\triangle), \quad$ and pMAPTAC $_{62}-$ DEAEMA $_{11}(\square)$ at $C_{\mathrm{p}}=10 \mathrm{~g} / \mathrm{L}$ in $0.1 \mathrm{M} \mathrm{NaCl}$ aqueous solutions.

and the diblock copolymers prepared by the RAFT process. In Figure 7, emission maxima for PNA fluorescence in the presence of the block copolymers are plotted as a function of $\mathrm{pH}$. For all the three block copolymers shown in Figure 7, the emission maxima are practically constant at $440 \mathrm{~nm}$ in a low $\mathrm{pH}$ regime $(\mathrm{pH} \leq 7)$, indicative of the absence of hydrophobic association of PNA with these block copolymers. The emission maxima for pMAPTAC $_{62}-$ DEAEMA $_{53}$ and pMAPTAC $_{62}-$ DEAEMA $_{28}$ shifted from $440 \mathrm{~nm}$ to 400 and $402 \mathrm{~nm}$, respectively in a narrow $\mathrm{pH}$ range of $7-8$ as $\mathrm{pH}$ was raised from an acidic $\mathrm{pH}$. This blue shift indicates a transfer of PNA from the aqueous phase to a hydrophobic environment formed by association of dehydrated DEAEMA blocks (i.e., micelle core). When $\mathrm{pH}$ was raised from 3 to 11 and subsequently reduced to 3 , completely reversible changes in the emission maximum without hysteresis were observed for both pMAPTAC $_{62}$-DEAEMA $_{53}$ and pMAPTAC $_{62}-\mathrm{DEAEMA}_{28}$. In the case of pMAPTAC $_{62}-$ DEAEMA $_{11}$, on the other hand, the blue shift of the emission maximum is much less than that of the other two block copolymers, the blue shift saturating at $413 \mathrm{~nm}$ at $\mathrm{pH} \geq 10$. These observations are qualitatively consistent with the light scattering data (Figure 5). For pMAPTAC $62-$ DEAEMA $_{11}$ the deprotonated DEAEMA block may not be hydrophobic enough, relative to the much longer MAPTAC block, to form a micelle core, although it provides a hydrophobic microenvironment where PNA probes are taken up to some extent at $\mathrm{pH}>7$.

\section{CONCLUSIONS}

A methacrylamide-based cationic monomer, MAPTAC, was first polymerized in the presence of 4-cyanopentanoic acid dithiobenzoate in water. The polymerization proceeded in accordance with a con- 
trolled mechanism that was confirmed from the observation that $M_{\mathrm{n}}$ increased linearly with monomer conversion while $M_{\mathrm{w}} / M_{\mathrm{n}}$ remained to be $c a$. 1.0 independent of the conversion. A dithioester-capped MAPTAC homopolymer thus prepared was used as a macroCTA, and block copolymers with DEAEMA were prepared in aqueous media. The block copolymerization of DEAEMA using the MAPTAC macro-CTA was confirmed to proceed in accordance with a "living" polymerization mechanism. The pMAPTAC $_{m}-$ DEAEMA $_{n}$ block copolymers exhibited $\mathrm{pH}$-induced micelle formation and disruption, which was investigated by ${ }^{1} \mathrm{H}$ NMR, QELS, SLS, and fluorescence techniques. These data indicated that pMAPTAC $_{62}-$ DEAEMA $_{53}$ and pMAPTAC $_{62}-$ DEAEMA $_{28}$ formed polymer micelles in $0.1 \mathrm{M} \mathrm{NaCl}$ aqueous solution at $\mathrm{pH}>7$. The $\mathrm{pH}$-induced formation and dissociation of micelle was completely reversible without hysteresis. There was a clear tendency that the number of polymer chains forming one micelle increases as the DEAEMA block length is increased.

\section{REFERENCES}

1. M. K. Georges, R. P. N. Veregin, P. M. Kazmaier, and G. K. Hamer, Macromolecules, 26, 2987 (1993).

2. M. K. Georges, R. P. N. Veregin, P. M. Kazmaier, and G. K. Hamer, Trends Polym. Sci., 2, 66 (1994).

3. G. Moad, A. G. Anderson, F. Ercole, C. H. J. Johnson, J. Krstina, C. L. Moad, E. Rizzardo, T. H. Spurling, and S. H. Thang, ACS Symp. Ser., 685, 332 (1998).

4. J. S. Wang and K. Matyjaszewski, Macromolecules, 28, 7901 (1995).

5. M. Sawamoto and M. Kamigaito, Trends Polym. Sci., 4, 371 (1996).

6. J. Chiefari, Y. K. Chong, F. Ercole, J. Krstina, J. Jeffery, T. P. T. Le, R. T. A. Mayadunne, G. F. Meijs, C. L. Moad, G. Moad, E. Rizzardo, and S. H. Thang, Macromolecules, 31, 5559 (1998).

7. D. Li and W. J. Brittain, Macromolecules, 31, 3852 (1998).

8. D. Benoit, V. Chaplinski, R. Braslau, and C. J. Hawker, J. Am. Chem. Soc., 121, 3904 (1999).

9. M. Teodorescu and K. Matyjaszewski, Macromol. Rapid Commun., 21, 190 (2000).

10. M. Senoo, Y. Kotani, M. Kamigaito, and M. Sawamoto, Macromolecules, 32, 8005 (1999).

11. Y. K. Chong, T. P. T. Le, G. Moad, E. Rizzardo, and S. H. Thang, Macromolecules, 32, 2071 (1999).

12. F. Ganachaud, M. J. Monterio, R. G. Gilbert, M.-A. Dourges, S. H. Thang, and E. Rizzardo, Macromolecules, 33, 6738 (2000).

13. M. S. Donovan, T. A. Sanford, A. B. Lowe, B. S. Sumerlin, Y. Mitsukami, and C. L. McCormick, Macromolecules, 35, 4570 (2002).

14. M. S. Donovan, A. B. Lowe, B. S. Sumerlin, and C. L. McCormick, Macromolecules, 35, 4123 (2002).

15. D. B. Thomas, B. S. Sumerlin, A. B. Lowe, and C. L.
McCormick, Macromolecules, 36, 1436 (2003).

16. B. S. Sumerlin, M. S. Donovan, Y. Mitsukami, A. B. Lowe, and C. L. McCormick, Macromolecules, 34, 6561 (2001).

17. K. Kataoka, G. S. Kwon, M. Yokoyama, T. Okano, and Y. Sakurai, J. Controlled Release, 24, 119 (1993).

18. L. M. Qi, H. Cölfen, and M. Antonietti, Angew. Chem. Int. Ed., 39, 604 (2000).

19. D. Oupicky, C. Konak, K. Ulbrich, M. A. Wolfert, and L. W. Seymour, J. Controlled Release, 65, 149 (2000).

20. M. Arotçaréna, B. Heise, S. Ishaya, and A. Laschewsky, J. Am. Chem. Soc., 124, 3787 (2002).

21. S. Yusa, Y. Shimada, Y. Mitsukami, T. Yamamoto, and Y. Morishima, Macromolecules, 36, 4208 (2003).

22. B. S. Sumerlin, A. B. Lowe, D. B. Thomas, and C. L. McCormick, Macromolecules, 36, 5982 (2003).

23. Y. Mitsukami, M. S. Donovan, A. B. Lowe, and C. L. McCormick, Macromolecules, 34, 2248 (2001).

24. Y. A. Vasilieva, D. B. Thomas, P. E. Hennaux, and C. L. McCormick, Polym. Prepr., Am. Chem. Soc., Div. Polym. Div., 44, 886 (2003).

25. A. J. Convertine, B. S. Sumerlin, D. B. Thomas, A. B. Lowe, and C. L. McCormick, Macromolecules, 36, 4679 (2003).

26. V. Bütün, N. C. Billingham, and S. P. Armes, Chem. Commun., 671 (1997).

27. A. S. Lee, A. P. Gast, V. Bütün, and S. P. Armes, Macromolecules, 32, 4302 (1999).

28. V. Bütün, S. P. Armes, and N. C. Billingham, Macromolecules, 34, 1148 (2001).

29. S. Liu, J. V. M. Weaver, Y. Tang, N. C. Billingham, S. P. Armes, and K. Tribe, Macromolecules, 35, 6121 (2002).

30. T. G. H. Yuen, W. F. Agnew, and L. A. Bullara, Biomaterials, 8, 138 (1987).

31. K. Srikulkit and P. Larpsuriyakul, Color. Technol., 118, 79 (2002).

32. M. Vamvakaki, N. C. Billingham, and S. P. Armes, Macromolecules, 32, 2088 (1999).

33. K. Mikkelsen and S. O. Nielsen, J. Phys. Chem., 64, 632 (1960).

34. J. Jakes, Czech. J. Phys., B38, 1035 (1988).

35. K. Schillén, W. Brown, and R. M. Johnsen, Macromolecules, 27, 4825 (1994).

36. B. H. Zimm, J. Chem. Phys., 16, 1099 (1948).

37. Y. A. Vasilieva, D. B. Thomas, C. W. Scales, and C. L. McCormick, Macromolecules, 37, 2728 (2004).

38. C. Bonnans-Plaisance, J. C. Gressier, G. Levesque, and A. Mahjoub, Bull. Soc. Chim. Fr., 891 (1985).

39. V. Bütün, S. P. Armes, and N. C. Billingham, Polymer, 42, 5933 (2001).

40. S. Pispas, N. Hadjichristidis, I. Potemkin, and A. Khokhlov, Macromolecules, 33, 1741 (2000).

41. S. Keki, G. Deak, A. Kuki, and M. Zsuga, Polymer, 39, 6053 (1998).

42. K. Kalyanasundaram and J. K. Thomas, J. Am. Chem. Soc., 99, 2039 (1977).

43. P. Overath and H. Träuble, Biochemistry, 12, 2625 (1973).

44. H. Träuble and P. Overath, Biochim. Biophys. Acta, 307, 491 (1973).

45. W. O. McClure and G. M. Edelman, Biochemistry, 5, 1908 (1966). 